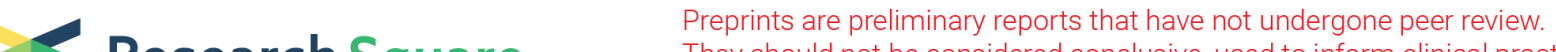 Research Square They should not be considered conclusive, used to inform clinical practice, or referenced by the media as validated information.
}

\section{Refractory vaccine-induced thrombotic thrombocytopenia (VITT) managed with delayed therapeutic plasma exchange (TPE)}

Ajay Major ( $\square$ ajay.major@uchospitals.edu )

The University of Chicago https://orcid.org/0000-0001-7261-1335

Timothy Carll

The University of Chicago

Clarence W. Chan

The University of Chicago

Chancey Christenson

The University of Chicago

Geoffrey D. Wool

The University of Chicago

Kenneth S. Cohen

The University of Chicago

\section{Case Report}

Keywords: coronavirus, platelet factor, thrombocytopenia, thrombosis, vaccines

Posted Date: July 16th, 2021

DOI: https://doi.org/10.21203/rs.3.rs-723623/v1

License: (c) (i) This work is licensed under a Creative Commons Attribution 4.0 International License.

Read Full License 


\section{Abstract}

Vaccine-induced thrombotic thrombocytopenia (VITT) is a newly-described hematologic disorder which presents as acute thrombocytopenia and thrombosis after administration of adenovirus-based vaccines against COVID-19. Due to positive assays for antibodies against platelet factor 4 (PF4), VITT is managed similarly to autoimmune heparin-induced thrombocytopenia (HIT) with intravenous immunoglobulin (IVIG) and non-heparinoid anticoagulation. We describe a case of VITT in a 50-year-old man with antecedent alcoholic cirrhosis who presented with platelets of $7 \times 10^{3} / \mu \mathrm{L}$ and portal vein thrombosis 21 days following administration of the Ad26.COV2.S COVID-19 vaccine. The patient developed progressive thrombosis and persistent severe thrombocytopenia despite IVIG, rituximab and high-dose steroids and had persistent anti-PF4 antibodies over 30 days after his initial presentation. As such, delayed therapeutic plasma exchange (TPE) was pursued as salvage therapy, with a rapid and sustained improvement in his platelet count. Our case serves as proof-of-concept of the efficacy of TPE in VITT.

\section{Introduction}

Vaccine-induced thrombotic thrombocytopenia (VITT) is a newly-described prothrombotic state identified in a small subset of patients following administration of adenovirus-based vaccines against COVID-19, such as the ChAdOx1 nCov-19 (AstraZeneca) and Ad26.COV2.S (Johnson \& Johnson) vaccines. Most patients affected by VITT present within 30 days of vaccination with acute thrombocytopenia and thrombosis, including unusual thromboses such as cerebral sinus venous thrombosis and arterial thrombosis. [1]

In early case series of VITT, nearly all patients exhibited strongly positive enzyme-linked immunosorbent assays (ELISA) for antibodies against platelet factor 4 (PF4) in the presence of a polyanion. [1-3] Given the combination of thrombosis, thrombocytopenia, and PF4 ELISA positivity in VITT patients, the disease resembles 'spontaneous' or autoimmune heparin-induced thrombocytopenia (HIT). [4-6] As such, the management of VITT mirrors the treatment paradigm of autoimmune HIT, with empiric initiation of nonheparinoid anticoagulation for thrombosis treatment as well as intravenous immunoglobulin (IVIG). [7, 8] Some consensus guidelines discuss that therapeutic plasma exchange (TPE) may be utilized for severe VITT, [9] although the role of TPE in HIT is controversial. [10]

We present a case of VITT refractory to multiple doses of IVIG, rituximab and high-dose steroids that was successfully managed with delayed TPE over 30 days after initial presentation. This case serves as a proof-of-concept that TPE may be used to remove anti-PF4 antibodies from the plasma compartment to improve the platelet count in refractory disease.

\section{Case Report}

A 50-year-old man with alcoholic cirrhosis and obesity presented to a local hospital with 1 week of generalized abdominal pain and distention, fatigue, and dark urine. In the emergency department, initial 
laboratory testing revealed platelets of $7 \times 10^{3} / \mu \mathrm{L}$, white blood cell count $9.5 \times 10^{3} / \mu \mathrm{L}$, hemoglobin 15.7 $\mathrm{g} / \mathrm{dL}$, INR 2.0, creatinine $1.1 \mathrm{mg} / \mathrm{dL}$, total bilirubin $2.2 \mathrm{mg} / \mathrm{dL}$, AST $235 \mathrm{U} / \mathrm{L}$, and ALT $198 \mathrm{U} / \mathrm{L}$. D-dimer was elevated at $23.64 \mathrm{mg} / \mathrm{L} \mathrm{FEU}$ and fibrinogen was normal at $211 \mathrm{mg} / \mathrm{dL}$. Computed tomography (CT) imaging revealed cirrhotic liver disease, increasing ascites, and possible thrombi in the portal and hepatic veins. He did not have recent heparin exposure. His baseline platelet count was $113-161 \times 10^{3} / \mu \mathrm{L}$ in the 6 months prior to admission. He had received the Ad26.COV2.S COVID-19 vaccine 21 days prior to presentation.

Given the concern for VITT, PF4 antibody testing and abdominal Doppler ultrasound were ordered. Abdominal ultrasonography confirmed complete thrombosis of the right portal vein and partial thrombus in the main portal vein. IgG-specific PF4/polyanion antibody ELISA testing (LIFECODES, Immucor) was positive at $1.578 \mathrm{OD}$. The patient was formally diagnosed with VITT and began treatment with argatroban and IVIG on the day of presentation.

The patient's platelet trend and VITT treatments over his hospital course are depicted in Fig. 1. The patient received IVIG $1 \mathrm{~g} / \mathrm{kg}$ on days 1 and 2 of admission with $125 \mathrm{mg}$ of intravenous methylprednisolone on day 2. Due to persistent thrombocytopenia, oral prednisone was initiated on day 7 at $120 \mathrm{mg}$ daily on a prolonged taper, with a small but transient increase in his platelet count to $21 \times 10^{3} /$ $\mu \mathrm{L}$ by day 12 . However, on day 14 , he developed progressive hepatic encephalopathy and asterixis concerning for acute liver failure. Repeat abdominal Doppler ultrasound on day 15 revealed progression of his portal vein thrombosis despite therapeutic argatroban.

On day 16 , the patient was transferred to our medical center for consideration of orthotopic liver transplantation. After arrival, the patient was switched to intravenous bivalirudin for anticoagulation and ADAMTS13 functional testing confirmed normal activity. Due to worsening thrombocytopenia to $9 \times 10^{3} /$ $\mu \mathrm{L}$ despite IVIG and high-dose steroids, the patient received his first dose of rituximab on day 17 , followed by two additional doses of IVIG $1 \mathrm{~g} / \mathrm{kg}$. P-selectin expression assay (Versiti, Inc.) was performed on day 19 and was negative, with $8 \%$ activity with $30 \mathrm{mcg}$ of PF4 and $0 \%$ activity with $30 \mathrm{mcg}$ of PF4 and $100 \mathrm{U}$ of heparin. The patient had a robust but again transient response to IVIG, with a maximum platelet count of $72 \times 10^{3} / \mu \mathrm{L}$ on day 24 . On day 24 , the patient underwent a transjugular intrahepatic portosystemic shunt (TIPS) procedure, with successful recanalization of the portal vein.

As shown in Fig. 1, the patient's platelet count continued to downtrend after day 24. A single unit of platelets was given on day 26 to determine if the patient was platelet-responsive, but there was no response and the patient reached a nadir of $24 \times 10^{3} / \mu \mathrm{L}$ on day 31 . A repeat CT of the abdomen on day 31 demonstrated an intact TIPS with complete resolution of the previous portal vein thrombosis. With no other apparent causes of persistent thrombocytopenia, refractory VITT was considered and a repeat antiPF4/polyanion ELISA (polyspecific IgG/IgA/IgM, LIFECODES, Immucor) on day 32 was positive at 2.063 OD. Given a persistently positive ELISA, the decision was made to pursue TPE. 
TPE was performed using the Spectra Optia (TerumoBCT) instrument via an internal jugular dialysis catheter. In each procedure, $6.0 \mathrm{~L}$ of plasma (equivalent to $\sim 1.1$ times the patient's total plasma volume) was exchanged using $100 \%$ donor plasma as replacement fluid with intravenous calcium gluconate.

After the first TPE on day 32, the polyspecific anti-PF4/polyanion ELISA was reduced to 1.010 OD. Given persistent thrombocytopenia, the decision was made to pursue serial TPE until the PF4 became negative. As shown in Fig. 1, the PF4 decreased to 0.452 OD after the second TPE on day 36, and then decreased to negative at 0.347 OD after the third TPE on day 37. TPE was generally uncomplicated, apart from a febrile non-hemolytic reaction after the second TPE that did not recur with premedication.

The patient had a robust improvement in his platelet count after the three TPE procedures, with a platelet count rising to $57 \times 10^{3} / \mu \mathrm{L}$ by day 42 (Fig. 1). A repeat PF4 ELISA on day 42 continued to be negative at 0.267 OD. As the patient's clinical status had significantly improved after the TIPS procedure, with resolution of hepatic encephalopathy and improvement in liver function, the patient was switched to subcutaneous fondaparinux for anticoagulation and was discharged from the hospital on day 44 with a platelet count of $68 \times 10^{3} / \mu \mathrm{L}$.

At outpatient hematology follow-up on day 49 after presentation, the patient's platelet count had recovered to $124 \times 10^{3} / \mu \mathrm{L}$, which is similar to his pre-VITT baseline.

\section{Discussion}

VITT is a novel and rare hematologic disease associated with COVID-19 vaccination. The pathophysiology of VITT is favored to mimic that of autoimmune HIT, [2] and similar antiplatelet immune thrombocytopenic-mediated thrombotic disorders have been described in patients receiving other polyanionic drugs. [11] Although therapies for HIT are implemented in VITT owing to this presumed similar etiology, the optimal management of VITT is unknown. In this case, typical HIT therapies were unsuccessful in halting progressive thrombosis and thrombocytopenia, necessitating the use of delayed TPE as salvage therapy.

Despite immediate initiation of IVIG, argatroban and high-dose steroids as recommended by consensus guidelines, [7-9] the patient developed progressive portal vein thrombosis and persistent severe thrombocytopenia by day 15 of admission. The finding of a negative P-selectin expression assay (PEA) on day 19 of admission was initially reassuring for resolution of VITT, as confirmatory testing using functional platelet activation assays, including serotonin release assay (SRA), heparin-induced platelet aggregation assay (HIPA), and PEA are frequently positive in VITT. [12] However, the presence of persistent thrombocytopenia $<30 \times 10^{3} / \mu \mathrm{L}$, which only transiently improved after repeat doses of IVIG and rituximab, prompted another assessment of the PF4 ELISA on day 32, which was markedly elevated and led to initiation of TPE. As shown in Fig. 1, three TPE procedures were sufficient to revert the PF4 ELISA to negativity and to rapidly increase the platelet count. Although it is also plausible in this case that weekly rituximab began to take effect at approximately the same time as TPE, the apparent TPE- 
dependent decrease in the PF4 ELISA with each subsequent TPE procedure suggests TPE as the more likely cause of the increasing platelet count. In retrospect, the negative PEA assay at day 19 may have been due to temporary effects of initial IVIG and steroid treatment on circulating functional antibodies.

TPE has recently been proposed as a salvage therapy for VITT for removal of pathogenic anti-PF4 antibodies, [13] and case reports describe both the successful [14] and unsuccessful [15] use of TPE in VITT. Given the delayed use of TPE with our patient, this case is proof-of-concept that TPE can be safe and effective in the setting of refractoriness to IVIG and high-dose steroids, which are often initiated early and simultaneously in the disease course.

\section{Conclusions}

We present a case of VITT in a patient with antecedent risk factors for thrombosis, including cirrhosis and obesity, with progressive thrombosis and severe thrombocytopenia despite non-heparin anticoagulation, IVIG and high-dose steroids, necessitating TPE for clearance of persistent anti-PF4 antibodies and improvement in the platelet count. In patients with documented VITT, there should be a low threshold for initiation of TPE if refractory thrombocytopenia or progressive thrombosis is present after 5 days of other recommended therapies.

\section{Learning Points}

Vaccine-induced thrombotic thrombocytopenia (VITT) is managed similarly to heparin-induced thrombocytopenia (HIT) given positive assays for antibodies against platelet factor 4 (PF4). We describe a patient with refractory VITT with persistent anti-PF4 antibodies over 30 days after presentation that was successfully managed with delayed therapeutic plasma exchange (TPE). TPE may be a safe and effective therapy for VITT that is refractory to typical treatments for HIT.

\section{Declarations}

$\underline{\text { Acknowledgments }}$

None to declare.

\section{Financial Disclosures}

There is no funding to declare for this manuscript.

\section{$\underline{\text { Conflicts of Interest }}$}



A. Major has no conflicts of interest to declare.
T. Carll has no conflicts of interest to declare.
C. W. Chan has no conflicts of interest to declare.
C. Christenson has no conflicts of interest to declare.
G. D. Wool receives honoraria from Diagnostica Stago (Parsippany, NJ).
K. S. Cohen has no conflicts of interest to declare.

\section{Informed Consent}

Informed consent for the publication of this case report was obtained from the patient.

\section{Authorship Contributions}

A. Major conceptualized and wrote the manuscript, performed data analysis and interpretation, and provided final approval of the manuscript.

T. Carll performed data analysis and interpretation, provided critical revision of the intellectual content, and provided final approval of the manuscript.

C. W. Chan performed data analysis and interpretation, provided critical revision of the intellectual content, and provided final approval of the manuscript.

C. Christenson performed data analysis and interpretation, provided critical revision of the intellectual content, and provided final approval of the manuscript.

G. D. Wool performed data analysis and interpretation, provided critical revision of the intellectual content, and provided final approval of the manuscript.

K. S. Cohen performed data analysis and interpretation, provided critical revision of the intellectual content, and provided final approval of the manuscript. 
The authors declare that all data are available within the manuscript.

\section{References}

1. Scully M, Singh D, Lown R, Poles A, Solomon T, Levi M, et al. Pathologic Antibodies to Platelet Factor 4 after ChAdOx1 nCoV-19 Vaccination. New England Journal of Medicine. 2021;384(23):2202-11.

2. Greinacher A, Thiele T, Warkentin TE, Weisser K, Kyrle PA, Eichinger S. Thrombotic Thrombocytopenia after ChAdOx1 nCov-19 Vaccination. New England Journal of Medicine. 2021;384(22):2092-101.

3. Schultz NH, Sørvoll IH, Michelsen AE, Munthe LA, Lund-Johansen F, Ahlen MT, et al. Thrombosis and Thrombocytopenia after ChAdOx1 nCoV-19 Vaccination. New England Journal of Medicine. 2021;384(22):2124-30.

4. Poudel DR, Ghimire S, Dhital R, Forman DA, Warkentin TE. Spontaneous HIT syndrome post-knee replacement surgery with delayed recovery of thrombocytopenia: a case report and literature review. Platelets. 2017;28(6):614-20.

5. Greinacher A, Selleng K, Warkentin TE. Autoimmune heparin-induced thrombocytopenia. Journal of Thrombosis and Haemostasis. 2017;15(11):2099-114.

6. Nazy I, Sachs UJ, Arnold DM, McKenzie SE, Choi P, Althaus K, et al. Recommendations for the clinical and laboratory diagnosis of VITT against COVID-19: Communication from the ISTH SSC Subcommittee on Platelet Immunology. Journal of Thrombosis and Haemostasis. 2021;19(6):15858.

7. International Society on Thrombosis and Haemostasis (ISTH). The ISTH Releases Interim Guidance on Vaccine-Induced Immune Thrombotic Thrombocytopenia (VITT) [Internet]. International Society on Thrombosis and Haemostasis. 2021 [cited 2021 Jul1]. Available from: https://www.isth.org/news/561406/The-ISTH-Releases-Interim-Guidance-on-Vaccine-InducedImmune-Thrombotic-Thrombocytopenia-VITT-.htm

8. British Society for Haematology. Guidance produced by the Expert Haematology Panel (EHP) focused on Vaccine Induced Thrombosis and Thrombocytopenia (VITT) [Internet]. British Society for Haematology. 2021 [cited 2021 Jul1]. Available from: https://b-s-h.org.uk/about-us/news/guidanceproduced-by-the-expert-haematology-panel-ehp-focussed-on-vaccine-induced-thrombosis-andthrombocytopenia-vitt/

9. COVID-19: Management of Vaccine-Associated Thrombotic Syndromes [Internet]. Penn Medicine Center for Evidence-Based Practice. 2021 [cited 2021Jul1]. Available from: http://www.uphs.upenn.edu/cep/COVID/TTS\%20treatment\%20520.pdf

10. Onuoha C, Barton KD, Wong ECC, Raval JS, Rollins-Raval MA, Ipe TS, et al. Therapeutic plasma exchange and intravenous immune globulin in the treatment of heparin-induced thrombocytopenia: A systematic review. Transfusion. 2020;60(11):2714-36.

11. Rosenthal MA, Rischin D, McArthur G, Ribbons K, Chong B, Fareed J, et al. Treatment with the novel anti-angiogenic agent PI-88 is associated with immune-mediated thrombocytopenia. Annals of 
Oncology. 2002;13(5):770-6.

12. Oldenburg J, Klamroth R, Langer F, Albisetti M, von Auer C, Ay C, et al. Diagnosis and Management of Vaccine-Related Thrombosis following AstraZeneca COVID-19 Vaccination: Guidance Statement from the GTH. Hämostaseologie. 2021;41(03):184-9.

13. Rock G, Weber V, Stegmayr B. Therapeutic plasma exchange (TPE) as a plausible rescue therapy in severe vaccine-induced immune thrombotic thrombocytopenia. Transfusion and Apheresis Science. $2021 ; 103174$.

14. Al-Mayhani T, Saber S, Stubbs MJ, Losseff NA, Perry RJ, Simister RJ, et al. Ischaemic stroke as a presenting feature of ChAdOx1 nCoV-19 vaccine-induced immune thrombotic thrombocytopenia. Journal of Neurology, Neurosurgery \& Psychiatry. 2021.

15. Sangli S, Virani A, Cheronis N, Vannatter B, Minich C, Noronha S, et al. Thrombosis With Thrombocytopenia After the Messenger RNA-1273 Vaccine. Annals of Internal Medicine. 2021.

\section{Figures}

150

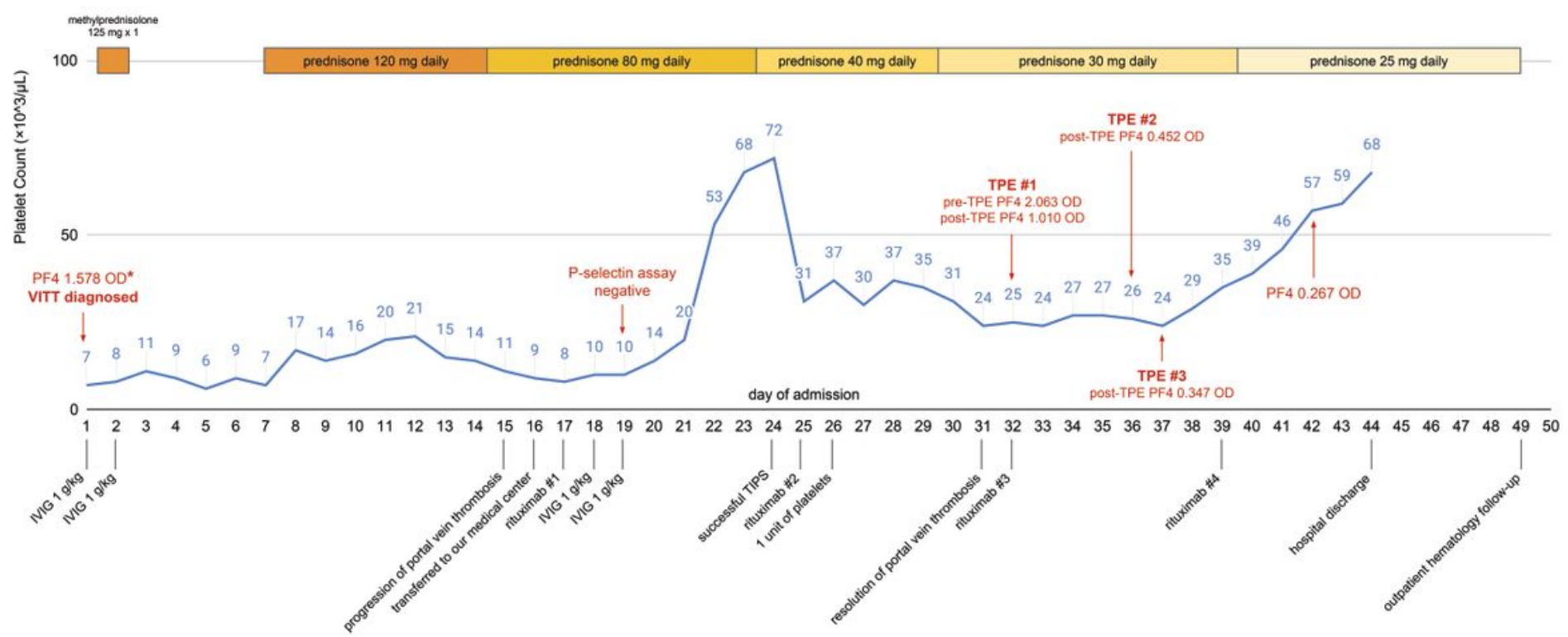

\section{Figure 1}

Platelet trend over the patient's hospital course, including all VITT testing and treatments. PF4 indicates anti-PF4/polyanion antibody ELISA testing. The asterisk indicates PF4/polyanion antibody ELISA testing performed at the outside hospital with an IgG-specific assay, whereas assays performed at our institution were performed with a polyspecific $\lg G / \lg \mathrm{A} / \lg M$ assay; as such, the optical density (OD) cannot be compared between these assays. 